\title{
READA: Redundancy Elimination for Accurate Data Aggregation in Wireless Sensor Networks
}

\author{
Kavi Khedo, Rubeena Doomun, Sonum Aucharuz \\ Faculty of Engineering, University of Mauritius, Réduit, Mauritius \\ E-mail:k.khedo@uom.ac.mu \\ Received January 14, 2010; revised February 20, 2010; accepted March 4, 2010
}

\begin{abstract}
In monitoring systems, multiple sensor nodes can detect a single target of interest simultaneously and the data collected are usually highly correlated and redundant. If each node sends data to the base station, energy will be wasted and thus the network energy will be depleted quickly. Data aggregation is an important paradigm for compressing data so that the energy of the network is spent efficiently. In this paper, a novel data aggregation algorithm called Redundancy Elimination for Accurate Data Aggregation (READA) has been proposed. By exploiting the range of spatial correlations of data in the network, READA applies a grouping and compression mechanism to remove duplicate data in the aggregated set of data to be sent to the base station without largely losing the accuracy of the final aggregated data. One peculiarity of READA is that it uses a prediction model derived from cached values to confirm whether any outlier is actually an event which has occurred. From the various simulations conducted, it was observed that in READA the accuracy of data has been highly preserved taking into consideration the energy dissipated for aggregating the data.
\end{abstract}

Keywords: Sensor Networks, Data Aggregation, Data Compression, Event Detection, Redundancy Elimination

\section{Introduction}

Advancement in wireless communication technologies and low-cost wireless devices for networking has initiated the emergence of a new type of wireless network, called wireless sensor network (WSN). WSN is a network consisting of a large number of sensor nodes that are deployed in an ad hoc manner over an area. For example, hundreds of sensors are spread out in an external or internal environment to measure the temperature, light intensity, humidity, noise level or any other physical conditions in its locality. The attractive feature of all these sensor nodes is that they can be self-organised and interconnected to form a network over which they can do cooperative processing to accomplish tasks that they cannot do individually [1].

Wireless sensor networks are being used in many different applications, initially for military networks as well as in other areas like environment, health, habitat monitoring and commercial purposes. With the recent breakthrough of "MicroElectroMechanical Systems (MEMS)" technology [2] whereby sensors are becoming smaller and more versatile, WSN promises many new application areas in the future.

Data aggregation minimizes redundant data which in turn reduces the number of packets transmitted to the base station resulting in conservation of energy and bandwidth. Since data aggregation transmits only the useful or resultant information to the end point, the problem of network congestion, traffic implosion and overlap can be overcome [3]. In classic flooding, nodes do not modify their activities based on the amount of energy available to them at a given time. Data aggregation techniques make the nodes of the network to be "resource-aware", i.e. adapt their communication and computation to the state of their energy resources.

The main goal of data aggregation is to collect and 'combine' data in an energy efficient manner so that the lifespan of the WSN is maximised. The design of an efficient data aggregation algorithm is a challenging research problem. Several performance measures like network lifetime, data accuracy, false alarm, high data redundancy, latency and scalability need to be considered concurrently $[4,5]$.

\section{Related Works}

In this section, some important existing aggregation techniques are discussed and their underlying assump- 
tions and drawbacks are considered.

\subsection{Greedy Aggregation}

In greedy aggregation $[5,6]$, a tree is constructed to indicate the path from each sensor node to the sink. The shortest path linking a node to the sink is used as an initialization of the tree. Then, the shortest paths linking the remaining nodes to the current tree will be incrementally added to enlarge the tree.

With this technique, the packets will be aggregated as early as possible and the aggregated packet will be directly routed back to the sink. However, the efficiency of the greedy incremental method is entirely determined by the shortest path. The data transmission is not reliable since once the path is broken, a large region will be disconnected and will not be able to send information to the sink.

\subsection{Tiny Aggregation (TAG)}

Tiny Aggregation (TAG) by Madden et al. [7,8] is a generic aggregation service that executes efficiently simple declarative queries. It is based on aggregation trees. The principal advantage of TAG is its ability to dramatically decrease the amount of communication required to compute an aggregate versus a centralized aggregation approach. TAG can tolerate loss due to information about queries in partial state records. Lost nodes can reconnect by listening to other sensor's state records - not necessarily intended for them - as they flow up the tree.

Another advantage of the TAG approach is that usually each sensor is required to transmit only a single message per epoch, regardless of its depth in the routing tree. In the centralized (non TAG) case, as data converges towards the root, nodes at the top of the tree are required to transmit significantly more data than nodes at the leaves. Thus, their batteries are drained faster and the lifetime of the network is limited. TAG may be inefficient for dynamic topologies or link failures. Failures at intermediate nodes lead to a disconnected sub tree. In addition, as the topology changes, TAG has to reorganize the tree structure, which means high costs in terms of energy consumption and overhead.

\subsection{Hybrid Energy Efficient Distributed Clustering Approach (HEED)}

HEED is a protocol proposed by Younis et al. $[9,10]$. In this approach, efficient clusters are formed for maximizing network lifetime. The main assumption in HEED is the availability of multiple power levels at sensor nodes. However, HEED does not make assumptions about the distribution of nodes or about node location-awareness. Cluster head selection is based on the residual energy of each node and a secondary parameter which depends on the node proximity to its neighbors. The cost of a cluster head is defined as its average minimum reachability power (AMRP). AMRP is the average of the minimum power levels required by all nodes within the cluster range to reach the cluster head. AMRP provides an estimate of the communication cost. At every iteration of HEED, each node which has not been selected as a cluster head sets its probability $\mathrm{PCH}$ of becoming the cluster head as:

$$
P C H=\mathrm{C} * \text { Eresidual / Emax }
$$

where $C$ denotes the initial percentage of cluster heads (specified by the user);

Eresidual is the estimated current residual energy of the node;

Emax is its initial energy corresponding to a fully charged battery.

HEED objectives are to prolong network lifetime by distributing energy consumption. HEED cluster heads are well distributed regardless of how the nodes are distributed in the network. This helps in maintaining high path quality at the inter-cluster level. In HEED, cluster heads are randomly selected based on their residual energy. Therefore, HEED cannot guarantee optimal head selection in terms of energy since it uses the secondary parameter to resolve conflicts. The cluster head is determined by repeated iterations. Thus, it requires a complex algorithm to calculate the number of rounds of iterations. Also, inter cluster communication has not been considered in HEED.

\section{Problem Statement}

Nodes in a WSN sense the environment and send the collected data to the base station. Many nodes report similar readings as data in a WSN are correlated. Thus, a large amount of energy is spent during the transmission of thousands of redundant data. Furthermore, as nodes transmit sensed values to the base station by transiting through intermediate nodes, significant energy is spent in communication. One technique used to decrease the number of redundant messages transmitted and thus prolong the network lifetime is data aggregation. During data aggregation, intermediate nodes merge the data received from other nodes into a single representative value. The energy of the network is conserved through a reduction in the number of messages being exchanged among nodes. However, in many current data aggregation techniques, a significant number of redundant messages still reach the base station. The lifetime of the sensor network is constantly faced by stringent energy consumptions. Other data aggregation techniques have been able to decrease the number of transmissions but at the 
cost of accuracy of final aggregates. Furthermore, if an uncorrelated (outlier) reading is sensed by a node, most data aggregation algorithms discard the outlier. The accuracy of the aggregate is again distorted by discarding such data. So far, no existing technique has been able to attenuate the limitations of data aggregation, explaining the wide research undertaken in this field.

\section{Proposed Data Aggregation System}

For the proposed data aggregation technique, the nodes will be organised into clusters where one node in each cluster will act as the clusterhead. Using the advantageous converging features of tree-based approach, inside each cluster the nodes will be arranged in a tree structure where the clusterhead will be the root.

This section introduces a new data aggregation technique called "Redundancy Elimination for Accurate Data Aggregation" (READA). The first part briefly describes the system design. The second part explains how data aggregation is performed in the monitoring system and in the event detection system. The last part describes the energy model that has been considered for designing the technique.

\subsection{System Model}

A number of sensor nodes $(\mathrm{N})$ are uniformly and randomly distributed in an area as shown schematically in Figure 1. In the simulation environment, this can be achieved by randomly assigning an $\mathrm{X}$-coordinate and $\mathrm{Y}$-coordinate in an area of $\mathrm{R} \times \mathrm{R} \mathrm{m}^{2}$. It is assumed that all node in the network (as well as the clusterhead) to be stationary and quasi-stationary. After the sensor nodes have been uniformly deployed over the sensor network field, the first stage of the algorithm is to form the clusters. A cluster protocol HEED as discussed in Section 2.3 is used for the cluster formation. The parameter used to choose a clusterhead $(\mathrm{CH})$ is based on the node's

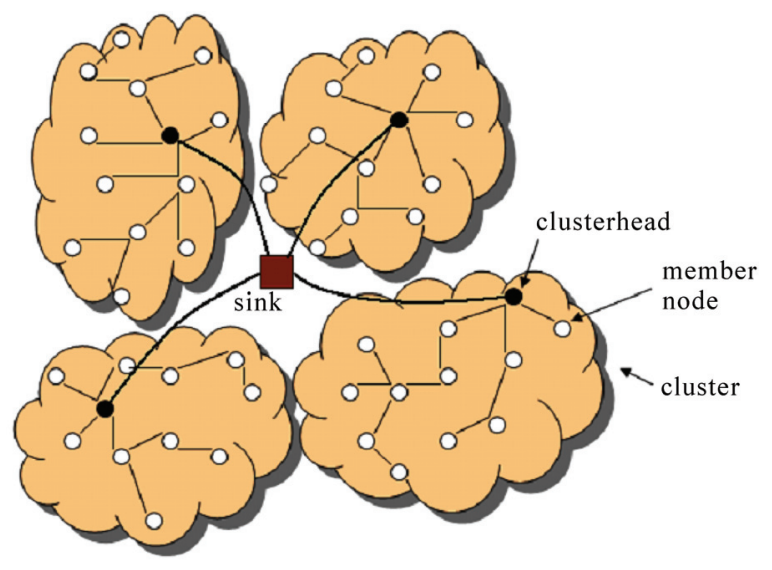

Figure 1. Network structure. residual energy. The node with highest residual energy among the tentative clusterheads is chosen as clusterhead $(\mathrm{CH})$. Once selected, the clusterhead advertises its status to all nodes in the cluster. The clusterhead needs not be necessarily in the centre of a cluster. The clusterhead can be positioned anywhere in the cluster, and it is assumed that all nodes in a cluster have a least a route to reach the clusterhead. If two or more nodes have the same highest residual energy, then randomly one of them is selected as the clusterhead.

The HEED clustering algorithm is run every $\mathrm{T}$ unit of time (or after $\mathrm{T}$ number of transmissions), where $\mathrm{T}$ generally depends on the type of application and initial battery energy of nodes. Each T unit of time is divided into $M$ rounds. In each round, a clusterhead receives observed data from nodes within its cluster.

The next stage of READA is to construct a tree within each cluster. The tree is constructed so that the workload of aggregating data is shared by every parent node rather than the clusterhead doing all the jobs. The clusterhead is chosen as root and sets its level to zero. It then broadcast a message to its single hop neighbours. Upon receiving the message, the nodes set their parent ID to that of the ID of where they received the message, i.e., ID of root and the level is incremented by 1 . Then, they broadcast a message to their respective single hop neighbours. The process is repeated until all nodes in the cluster have a parent.

\subsection{Aggregation}

Aggregation will be performed for two types of events: monitoring system and event detection.

\section{Correlation coefficient}

Sensor nodes across a network field collect readings that span over a range of values. The larger the size of the network, the readings collected from different areas of the field span over larger ranges. Consider thousands of sensors deployed over hectares of forest land for fire detections as shown in Figure 2.

The nodes in the "red region" are spatially correlated, that is, close nodes detect similar (but not equal) values.

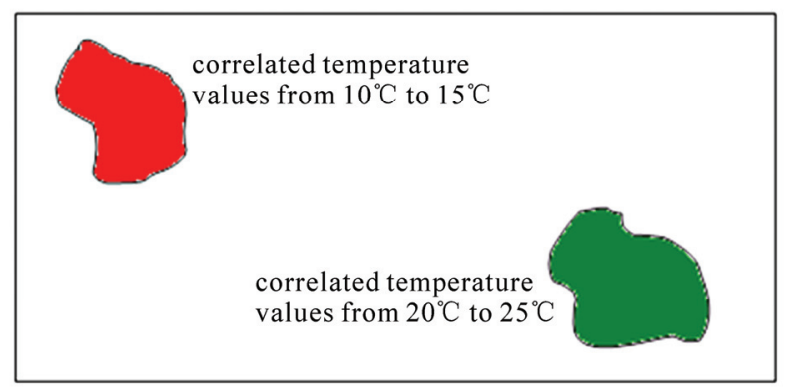

Figure 2. Different ranges of temperature value recorded over a large forest region. 
However, it should not be expected that nodes in the "green region", found thousands of kilometers away in the sensor field, collect values similar to that of the "red region". The temperature in the "red region" is due to one phenomenon while that of the "green region" is caused by other phenomenon. Studies have shown that correlation property of observed phenomenon is not always transitive [11], i.e., different phenomenon from the same set of nodes has different degree of correlation.

READA defines a single value called the correlation coefficient to represent the whole set of readings recorded by all the nodes in the sensor field. The value of the correlation coefficient $(\mathrm{H})$ ranges from 1 to $10 . \mathrm{H}=1$ is for strong correlation of data and as the correlation coefficient increases, the degree of correlation between data decreases. For instance, if in the above forest region the range of temperatures recorded is between $10^{\circ} \mathrm{C}$ and $20^{\circ} \mathrm{C}$, a correlation coefficient of 1 is assigned to the global set of readings. A larger range of values recorded, say from $20^{\circ} \mathrm{C}$ to $40^{\circ} \mathrm{C}$, is represented by a larger correlation coefficient of 2 . Also, the correlation coefficients assigned to the whole set of recorded value is application specific.

\subsubsection{Monitoring System}

A monitoring system is used to inspect a sensor field. It is assumed that $\mathrm{N}$ sensor nodes have identical characteristics deployed with high node density and no unexpected or drastic event occurs for short interval of times. Hence, the data $X_{i}$ sensed and recorded by any neighbouring nodes $\mathrm{i}(1<\mathrm{i}<\mathrm{N})$ can be assumed to exhibit high spatial correlation (i.e. data sensed by neighbouring nodes are similar) as well as good temporal correlation (i.e. data sensed by a specific node over time is comparable). Such data can be averaged to accurately represent all readings with a single value.

\subsubsection{Grouping}

Grouping is a technique used to partition data. It first selects the pivots, produced from grouping expressions, by which all readings will refer to in the aggregation process.

Let $\mathrm{V}_{\mathrm{i}}$ be the value of node $\mathrm{i}$,

Let $\mathrm{R}_{\mathrm{F}}$ be a user-defined redundancy factor,

Let $\mathrm{P}_{1}$ and $\mathrm{P}_{2}$ be the 2 group pivots formed by the grouping expression,

then if $\mathrm{V}_{\mathrm{i}} \leq \mathrm{P}_{1}$,

$\left|\mathrm{V}_{\mathrm{i}}-\mathrm{P}_{1}\right| \leq \mathrm{R}_{\mathrm{F}}$

$V_{i}$ forms part of group $P_{1}$ else $V_{i}$ forms part of group $P_{2}$.

The following diagram explains how aggregates are added to groups.

\section{Case 1}

A stream of data is used rather than a single value to better demonstrate how the values in the sensor field are changing gradually. From the final aggregate in Case 1, it can be deduced that the temperature recorded were $15^{\circ} \mathrm{C}, 22^{\circ} \mathrm{C}$ and $31^{\circ} \mathrm{C}$ as shown in Figure 3 .

Case 2

Based on the assumption of packet size being of 30 bytes, the aggregate produced can easily fit in a packet. More details about the phenomenon can be added which will make the aggregate more accurate. Each packet can also indicate the number of nodes that have participated to give this result as shown in Figure 4.

From the final aggregate in Case 2, the temperature

Case 1

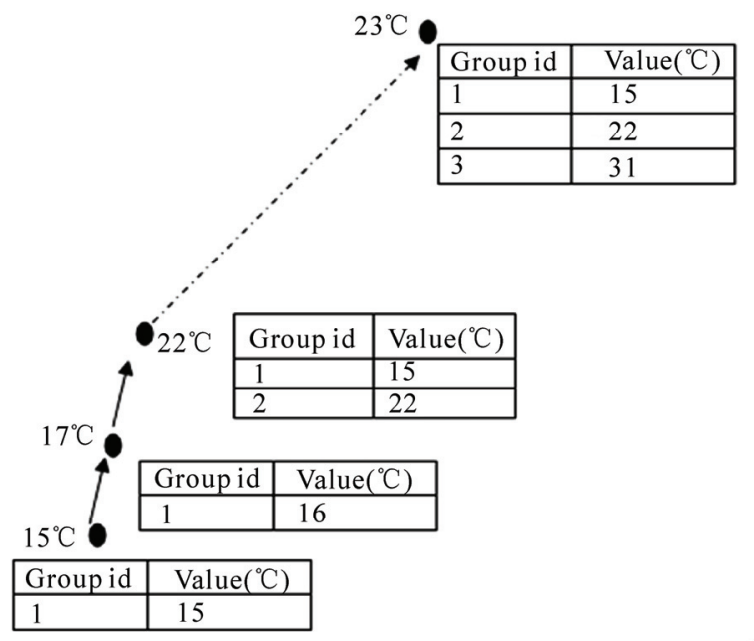

Figure 3. Case 1 aggregate grouping.

Case 2

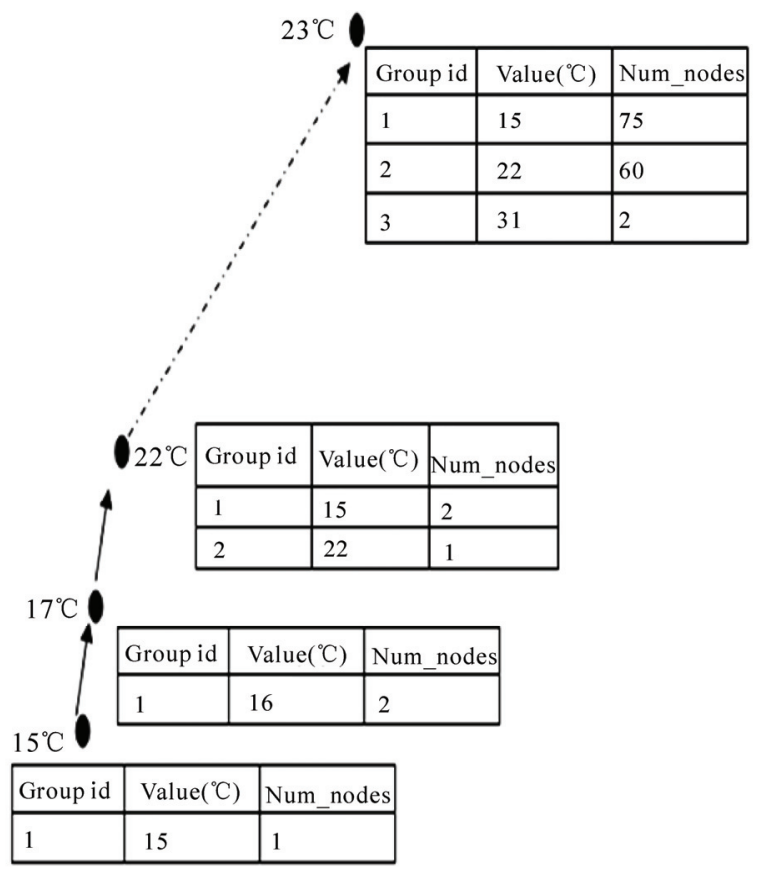

Figure 4. Case 2 aggregate grouping. 
recorded were $15^{\circ} \mathrm{C}, 22^{\circ} \mathrm{C}$ and $31^{\circ} \mathrm{C}$. It can also be seen that a larger number of nodes detected a reading of $15^{\circ} \mathrm{C}$ and $22^{\circ} \mathrm{C}$, while a temperature of $31^{\circ} \mathrm{C}$ was merely present among other nodes.

\subsubsection{Compression}

Since the group pivots are known by the base station, instead of representing the aggregate of each group by the actual value, it can be represented by a group ID. Furthermore, data in the group can be compressed instead of sending all data as shown in Figure 5.

Representation of a group:

[group ID | compressed value | Number of nodes participating]

Consider the following set of data A where pivot 1 is 10 with group ID $=1$, pivot 2 is 20 with group ID $=2$ and pivot 3 is 30 with group ID $=3$.

Knowing that energy utilisation is proportional to number of bits transmitted, the energy spent in sending the set $\mathrm{A}^{1}$ is much smaller than the energy spent in sending the set $\mathrm{A}$.

\subsubsection{Excess Groups}

Generally, sensor nodes are deficient in memory capacity, the amount of data that can be stored and transmitted is limited. In the design, for simulation purposes, it is assumed that no more than seven data groups can fit in the sensor node memory. This design assumption does not impact the simulation as a sensor node will only be required to communicate with its neighbours and its memory space will not be overloaded.

\section{Decision Point 1}

In order to maintain the accuracy and prevent the loss of data, information cannot be discarded. The strategy adopted is to merge groups into a single group while maintaining the accuracy of the aggregate. The choice of the groups to be merged is to look at the proximity between aggregates in neighbouring groups. READA analyses the groups which are closer to each other and

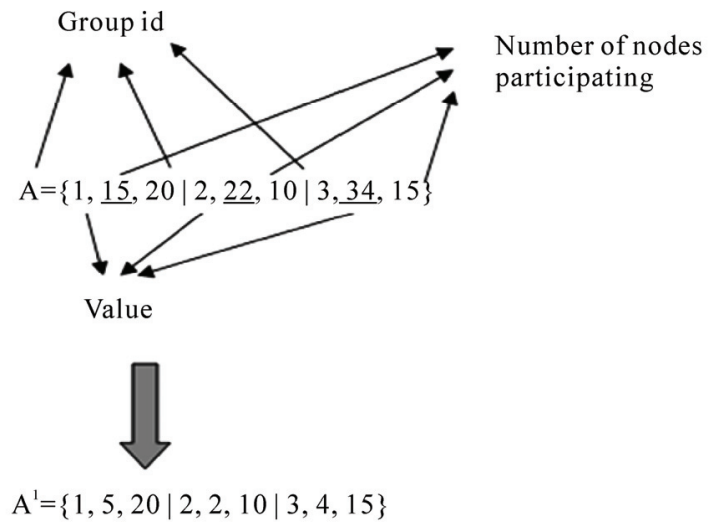

Figure 5. Compression. then merges those groups as illustrated in Figure $\mathbf{6}$ below.

Since the difference in the values of group 0 and group 1 is very small, it is best to merge these 2 groups into 1 group.

\section{Decision Point 2}

After the groups to be merged have been identified, it is necessary to identify which one of the two groups has to be evicted and which one has to be retained. READA determines the proximity of the first aggregate from each group and performs a weighted average. In the above diagram, since value 9 is closer to group 1 , group 1 is given a weight of 2 and group 0 is given a weight of 1 .

Thus, the final aggregate is as shown in Figure 7 below:

\subsubsection{Event Detection}

READA is basically a monitoring system where nodes can periodically switch to idle mode. Idle mode enables energy savings in sensor nodes that are neither transmitting nor receiving packets. However, READA can also behave as an event detection system where nodes continuously sense the environment and report if an unusual behaviour is noted. An event is defined as a different behaviour, i.e., which does not exhibit correlation over a similar region. An abnormal data sensed need not necessarily be an event as it might be a false alarm. In such situation, READA analyses the cached data of its neighbouring nodes, extrapolates their values to determine whether the abnormal data sensed was actually an event not a false alarm. The event is reported only if the
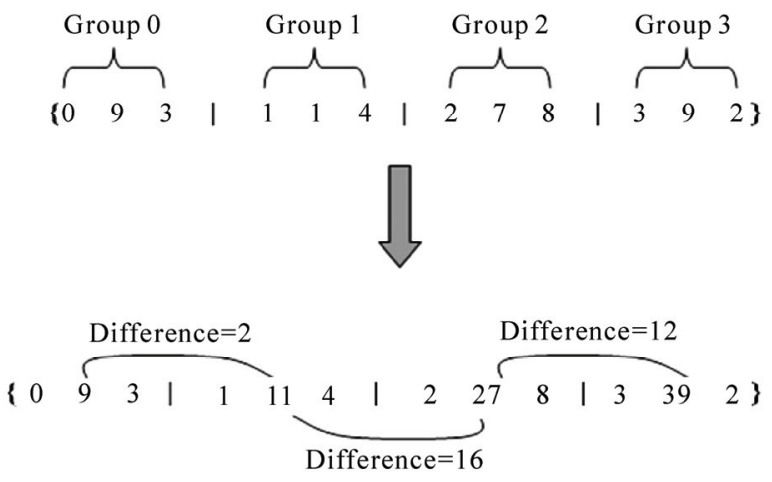

Figure 6. Merging groups.

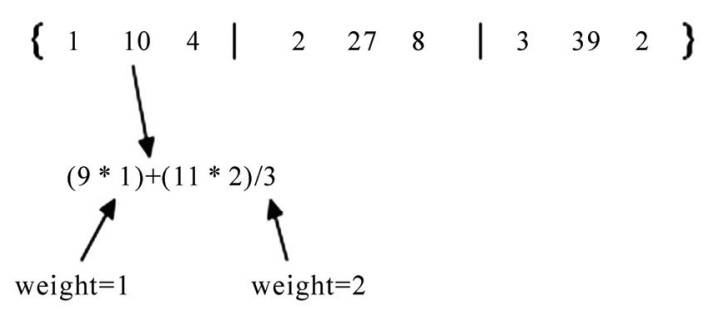

Figure 7. Final aggregate. 
probability of the occurrence of the event passes a userdefined confidence level.

\subsubsection{Outlier Detection}

Intermediate node analyses the set of results obtained from its child nodes together with its own to ensure there is spatial correlation among all the readings. The technique used is similar to the method used in Lightweight Temporal Compression (LTC) [12]. Since environmental data have a nice property that they are usually continuous in the temporal dimension and at small enough time windows are approximately linear, all the results can be continuously mapped onto a line. However, in the presence of an outlier the point can no longer fit on that line. The graphs below represent how the points are mapped onto the same line and how an outlier deviates from the mapped line of spatially correlated data.

In Case 1, the first point $S_{1}$ is set as a sample point. A highline and a lowline are chosen to represent data with a certain error margin, e, from the point $S_{2}$ as shown in Figure 8. The shaded area represents all the possible values that can fit between the two values. The next point fits between highline and lowline and thus, the next plotted value shows spatial correlation.

In Case 2, shown in Figure 9, there is the presence of outliers as the points do not fit in the correlation space. Hence, they cannot be plotted in the shaded region.

\subsubsection{Least Square Extrapolation}

In order to perform an extrapolation, READA requires the parent nodes to cache at least two previous values of their child nodes. A suitable extrapolation technique is

Case 1
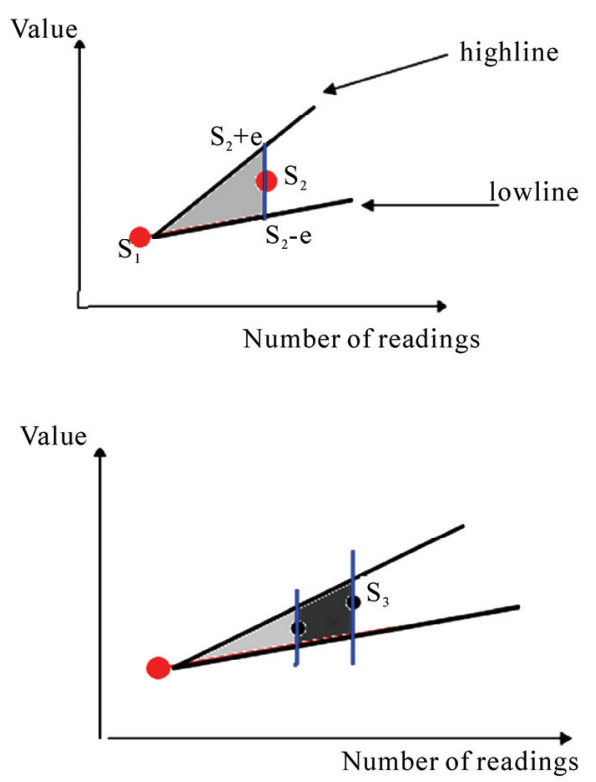

Figure 8. Case 1 outlier detection.

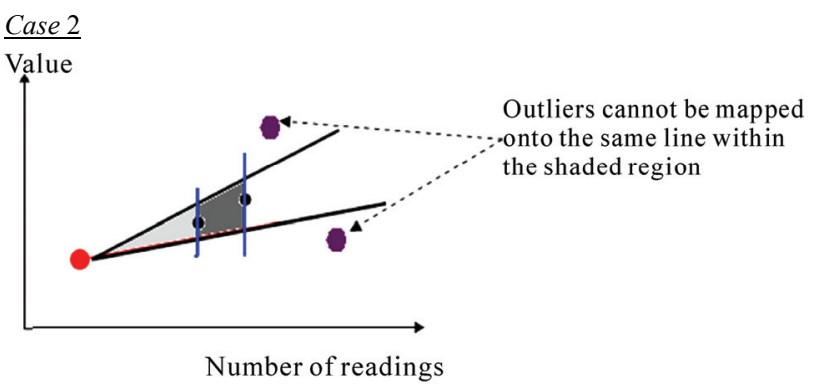

Figure 9. Case 2 outlier detection.

the Least Square Extrapolation as environmental variable tends to change linearly over time. The choice of Least Square is based on its low complexity which makes it appropriate for low battery power and memory deficient nodes compared to other extrapolation techniques.

The following formula is used to identify the trend line through the data:

$\gamma$, the trend for a given time period $=\mathbf{a}+\mathbf{b x}$ where

$$
\begin{aligned}
\mathrm{b} & =\frac{n \sum x y-\sum x \sum y}{n \sum x^{2}-\left(\sum x\right)^{2}} \\
\mathrm{a} & =\frac{\sum y}{n}-b \frac{\sum x}{n}
\end{aligned}
$$

$\boldsymbol{x}$ refers to the cached time values.

$\boldsymbol{y}$ refers to the cached values .

When an outlier has been detected, the parent node decides to make an extrapolation based on cached values. The aim of doing so is to predict the occurrence of an event. If the extrapolated value corresponds to a certain degree to the outlier, events can be identified. However, if the extrapolated value is overestimated or underestimated, the outlier is declared as a false alarm.

\section{Experimental Results and Evaluation}

In this section, simulations are carried out to demonstrate the performance of READA. Two other techniques are compared with READA to show its performance in terms of energy efficiency, accuracy and outlier detection. From the several simulation scenarios, the results are plotted graphically for analysis and evaluation.

READA will be compared with Conventional data aggregation which allows all unique sensed data to arrive at the base station. All sensor nodes collect data from the child nodes arranged in a tree like structure, concatenate the message with their own sensed data and forward the aggregate up the tree. Conventional data aggregation has been chosen to demonstrate how data aggregation affects the status of sensor networks. READA is also compared with another algorithm called Tiny Ag- 
gregation (TAG). TAG uses a grouping mechanism for aggregating data.

\section{Correlation Coefficient}

During the simulation, the data range is assumed to be in the set $[0 \ldots 100]$. Thus, ranges of data with different degree of correlation from this set are used. The correlation coefficient is defined as a measure of the spread of the set of data. For example $[0 \ldots 10]$, the correlation coefficient is $1,[0 \ldots 50]$ it is $5,[0 \ldots 100]$ it is 10 and so on.

\section{Accuracy}

The Mean Average Deviation (MAD) approach is used to determine accuracy of data. The accuracy in the simulations is expressed in terms of the average deviation of the estimated mean to the actual mean. A higher MAD value implies lower data accuracy.

$$
\begin{aligned}
& \text { Accuracy } \approx \\
& \frac{\mid \text { Estimated Mean - Actual Mean } \mid}{\text { Actual Mean }} \times 100
\end{aligned}
$$

\subsection{Energy Comparison}

A combined table of values obtained by READA, TAG and conventional is shown in Table $\mathbf{1}$ to better assess their energy efficiency.
The values and graphs in Table 1 and Figure 10 show that READA spends ranging from $23 \%$ to $27 \%$ less than Conventional method. This is for the obvious reason that Conventional method does not perform any aggregation. Hence READA has saved an average of $25 \%$ of energy in one round of the running of the data aggregation process.

The values and graphs in Table 1 and Figure 11 show that READA spends $(\sim 9 \%)$ more amount of energy than TAG when number of nodes is 500 (Run 1). However, when the number of nodes increases, the amount of energy dissipated is approximately the same as that of TAG. As the correlation coefficient increases, data become more uncorrelated and the energy consumed is higher as the packet size is greater. This is because both TAG and READA has a tree structure where they have to route up the data and this consumed energy because of greater exchange in number of bits transmissions.

\subsection{Accuracy Comparison}

A combined table of values obtained by READA and TAG is shown in Table 2 to better assess their accuracy performance.

\begin{tabular}{|c|c|c|c|c|c|c|c|c|c|c|c|c|c|c|c|}
\hline \multirow[b]{2}{*}{$\begin{array}{l}\text { Number } \\
\text { of nodes }\end{array}$} & \multicolumn{3}{|c|}{ Corr.Coeff $=1$} & \multicolumn{3}{|c|}{ Corr.Coeff $=2$} & \multicolumn{3}{|c|}{ Corr.Coeff $=3$} & \multicolumn{3}{|c|}{ Corr.Coeff $=4$} & \multicolumn{3}{|c|}{ Corr.Coeff $=5$} \\
\hline & TAG & READA & Con. & TAG & READA & Con. & TAG & READA & Con. & TAG & READA & Con. & TAG & READA & Con. \\
\hline 500 & 45.47 & 49.37 & 58.17 & 46.65 & 50.68 & 58.89 & 47.41 & 51.50 & 59.51 & 48.53 & 52.55 & 61.77 & 49.43 & 54.00 & 65.67 \\
\hline 1000 & 90.92 & 94.95 & 119.01 & 93.33 & 96.79 & 121.43 & 94.87 & 98.32 & 123.94 & 97.02 & 100.86 & 129.31 & 98.87 & 102.97 & 133.45 \\
\hline 1500 & 136.38 & 140.63 & 179.58 & 139.87 & 143.35 & 185.09 & 141.99 & 145.47 & 188.20 & 145.87 & 148.65 & 196.05 & 148.06 & 151.98 & 204.88 \\
\hline 2000 & 181.81 & 186.00 & 240.05 & 186.50 & 189.64 & 248.67 & 189.75 & 192.23 & 253.40 & 193.86 & 196.58 & 264.45 & 197.56 & 199.67 & 274.67 \\
\hline 2500 & 227.21 & 231.50 & 300.67 & 233.25 & 236.05 & 311.28 & 237.01 & 238.79 & 319.39 & 242.10 & 243.40 & 331.04 & 246.79 & 248.97 & 348.58 \\
\hline 3000 & 272.68 & 277.12 & 366.93 & 279.85 & 281.86 & 373.57 & 284.59 & 285.71 & 382.45 & 290.79 & 291.49 & 403.12 & 295.99 & 298.40 & 421.30 \\
\hline
\end{tabular}

Table 1. Combined energy.

Table 2. Combined accuracy.

\begin{tabular}{ccccccccccc}
\hline & \multicolumn{2}{c}{ Corr.Coeff $=1$} & \multicolumn{2}{c}{ Corr.Coeff $=2$} & \multicolumn{2}{c}{ Corr.Coeff $=3$} & \multicolumn{2}{c}{ Corr.Coeff $=4$} & \multicolumn{2}{c}{ Corr.Coeff $=5$} \\
\hline $\begin{array}{c}\text { Number } \\
\text { of nodes }\end{array}$ & TAG-1 & READA-1 & TAG-2 & READA-2 & TAG-3 & READA-3 & TAG-4 & READA-4 & TAG-5 & READA-5 \\
500 & 178 & 1.13 & 1.37 & 1.07 & 1.52 & 0.87 & 1.58 & 0.85 & 1.56 & 0.24 \\
1000 & 188 & 1.30 & 1.75 & 1.14 & 1.55 & 1.07 & 1.00 & 0.64 & 1.86 & 0.53 \\
1500 & 1.57 & 1.27 & 1.70 & 1.14 & 1.51 & 1.08 & 1.06 & 0.31 & 2.80 & 1.61 \\
2000 & 1.85 & 1.29 & 1.73 & 1.00 & 1.54 & 1.00 & 2.00 & 0.08 & 3.02 & 2.04 \\
\hline
\end{tabular}




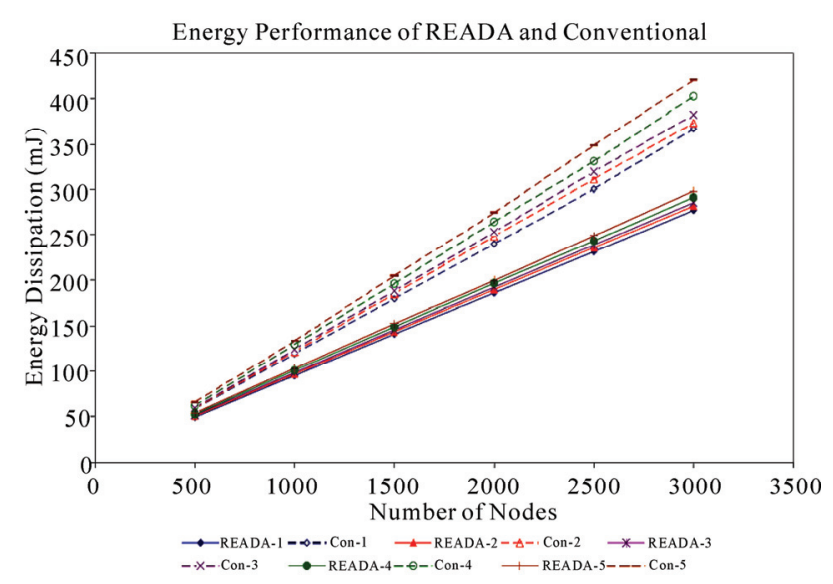

Figure 10. Energy performance of READA and Conventional technique.

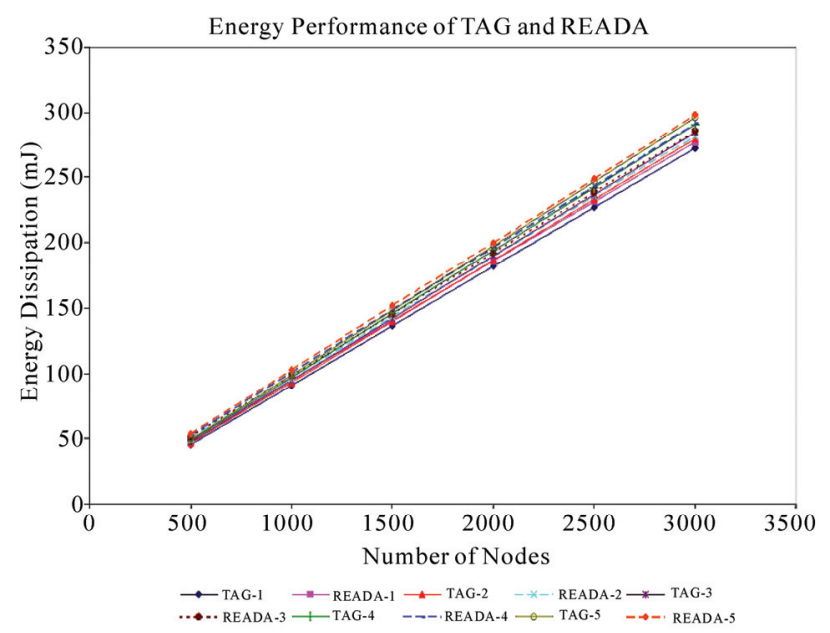

Figure 11. Energy performance of TAG and READA.

In all cases, READA has a smaller average deviation from mean, as shown in Figure 12 and Table 2. For the correlation coefficient 1, 2 and 3, READA outperforms TAG about $29-34 \%$ more accuracy than TAG. This shows that the grouping mechanism used in READA provides meaningful aggregate by keeping to a large extent the accuracy of the data. For correlation coefficient 4 and 5, READA has a technique of doing group retention while TAG does group eviction. This can explain why READA provides better accurate aggregate than TAG when the correlation coefficient is large. However, for correlation coefficient 5 despite doing group retention by READA, the deviation from mean show an increase trend. This is because of high uncorrelated data.

\subsection{Detection of False Messages Comparison}

A combined table of values obtained by READA and conventional technique is shown in Table 3 to better assess the aspect of recording the average number of false messages.

Conventional method treats false alarms and event equally. Through correct extrapolation, READA cuts down the number of false alarms by nearly $80 \%$ as shown in Figure 13.

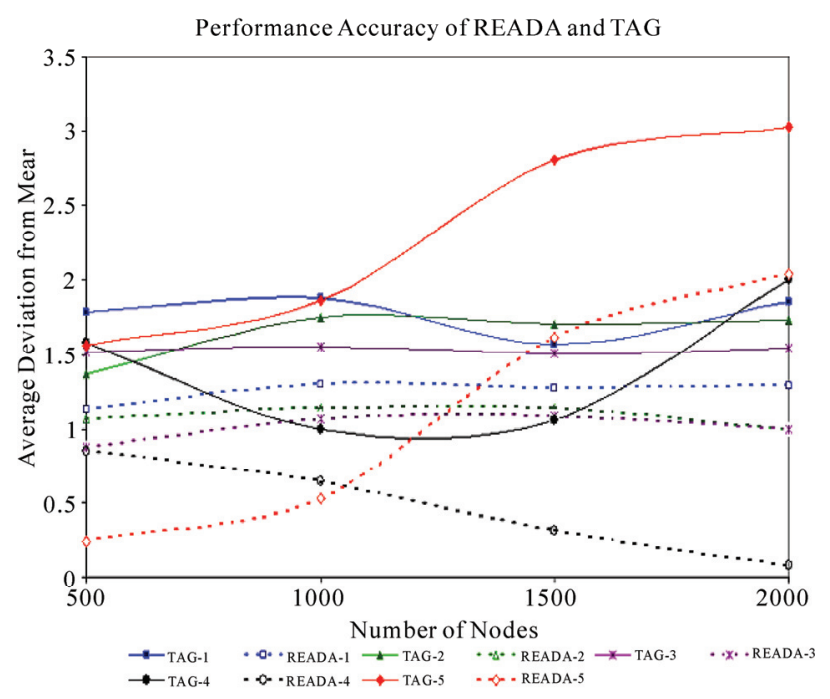

Figure 12. Accuracy performance of READA and TAG.

Table 3. Combined number of false messages recorded.

\begin{tabular}{ccccc}
\hline & \multicolumn{2}{c}{ Number of nodes is 40} & \multicolumn{2}{c}{ Number of nodes is 60} \\
\hline $\begin{array}{c}\text { Number of } \\
\text { Explosions }\end{array}$ & Con-40 & READA-40 & Con-60 & READA-60 \\
5 & 5 & 1 & 5 & 1 \\
6 & 6 & 0 & 6 & 1 \\
7 & 7 & 1 & 7 & 2 \\
\hline
\end{tabular}

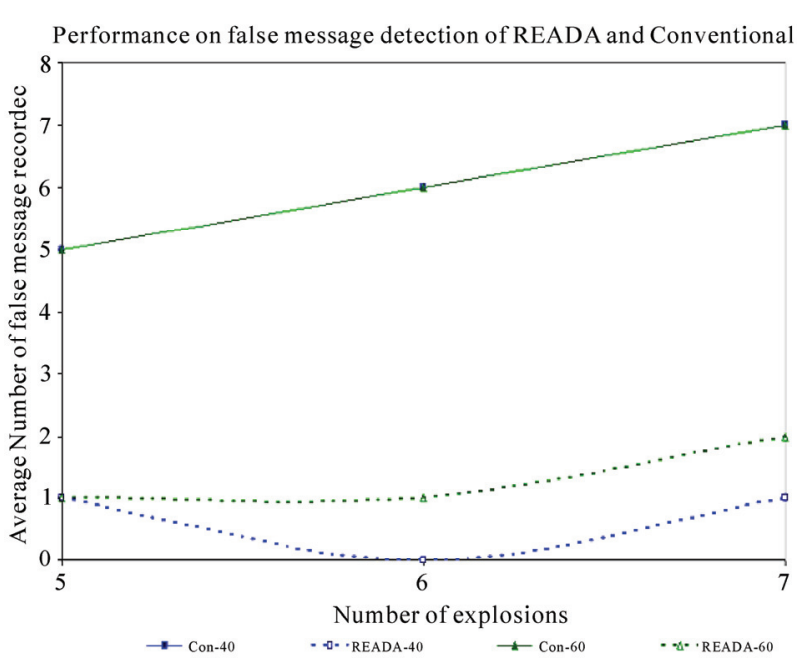

Figure 13. Number of false alarms. 


\section{Conclusions}

The strategy adopted by READA is a grouping and compression mechanism to produce a compressed but accurate aggregate. Since the set of data sensed exhibit high spatial correlation, READA partitions them into groups. A group consists of a group id, the compressed value and the number of nodes participating to give this compressed value. The group id is the pivot which has been determined by the base station. Since sensor nodes are deficient in memory capabilities, when there are an excess number of groups, the value in the two groups is merged based on the proximity of the aggregate in the two groups. The decision of group eviction and group retention is determined by performing a weighted average.

Another aspect of READA is outlier detection, that is, data that do not conform to the correlation space. Taking the advantage of the high degree of spatial correlations that exist among the sensor readings of the adjacent nodes in a densely deployed WSN, outliers can be detected if a prediction model is used. READA uses Least Square Extrapolation based on cached data to determine whether the outlier is actually an event that has occurred or a false alarm. From simulation results gathered, READA offers some $25 \%$ in terms of energy saved compared to conventional data aggregation and READA's accuracy was on average $40 \%$ more than TAG. It considerably outperforms TAG in terms of accuracy performance and outlier detection. READA can find out whether an outlier is a false alarm or an event that the base station needs to be informed about. Nearly $80 \%$ of false alarms can be filtered.

\section{References}

[1] I. F. Akyildiz, W. Su, Y. Sankarasubramaniam and E. Cayirci, "Wireless Sensor Networks: A Survey," Elservier Computer Networks, Vol. 38, No. 4, March 2002, pp. 393-422.

[2] B. Warneke and K. S. J. Pister, "MEMS for Distributed Wireless Sensor Networks," 9th International Conference on Electronics, Circuits and Systems, Dubrovnik, 15-18
September 2002.

[3] J. Kulik, W. R. Heinzelman and H. Balakrishnan, "Negotiation-Based Protocols for Disseminating Information in Wireless Sensor Networks," Wireless Networks, Vol. 8, March 2002, pp. 169-185.

[4] R. Rajagopalan and P. K. Varshney, "Data-Aggregation Techniques in Sensor Networks: A Survey," IEEE Communication Surveys and Tutorials, Vol. 8, No. 4, December 2006, pp. 48-63.

[5] X. Li, "A Survey on Data Aggregation in Wireless Sensor Networks,” Project Report for CMPT 765, Spring 2006.

[6] H. Albert, R. Kravets and I. Gupta, "Building Trees Based On Aggregation Efficiency in Sensor Networks," Ad Hoc Networks, Vol. 5, No. 8, November 2007, pp. 1317-1328.

[7] S. R. Madden, M. J. Franklin, J. M. Hellerstein and W. Hong, "TAG: Tiny Aggregation Service for Ad-Hoc Sensor Networks," Proceedings of the 5th symposium on Operating Systems Design and Implementation, Boston, December 2002.

[8] E. Fasolo, M. Rossi, J. Widmer and M. Zorzi, "In-network Aggregation Techniques for Wireless Sensor Networks: A Survey," IEEE Wireless Communications, Vol. 14, No. 2, April 2007, pp. 70-87.

[9] O. Younis and S. Fahmy, "HEED: A Hybrid, Energy Efficient, Distributed Clustering Approach for Ad-hoc Sensor Networks," Proceedings of IEEE Transactions on Mobile Computing, Vol. 3, No. 4, October-December 2004, pp. 366-379.

[10] R. Rajagopalan and P. K. Varshney, "Data-Aggregation Techniques in Sensor Networks: A Survey," IEEE Communication Surveys and Tutorials, Vol. 8, No. 4, December 2006, pp. 48-63.

[11] S. Tanachaiwiwat and A. Helmy, "Correlation Analysis for Alleviating Effects of Inserted Data in Wireless Sensor Networks," Proceedings of the 2nd Annual International Conference on Mobile and Ubiquitous Systems: Networking and Services, San Diego, July 2005, pp. 97- 108.

[12] T. Schoellhammer, B. Greenstein, E. Osterweil, M. Wimbrow and D. Estrin, "Lightweight Temporal Compression of Microclimate Datasets," 29th Annual IEEE International Conference on Local Computer Networks, Washington, DC, November 2004, pp. 516-524. 